\title{
A rapid decrease in the expression of DMT1 and Dcytb but not Iregl or hephaestin explains the mucosal block phenomenon of iron absorption
}

\author{
D M Frazer, S J Wilkins, E M Becker, T L Murphy, C D Vulpe, A T McKie, G J Anderson
}

Background: A large oral dose of iron will reduce the absorption of a subsequent smaller dose of iron in a phenomenon known as mucosal block. Molecular analysis of this process may provide insights into the regulation of intestinal iron absorption.

Aims: To determine the effect of an oral bolus of iron on duodenal expression of molecules associated with intestinal iron transport in rats and to relate this to changes in iron absorption.

See end of article for authors' affiliations Methods: Rats were given an oral dose of iron and duodenal expression of divalent metal transporter 1 (DMT 1), Dcytb, Ireg 1, and hephaestin ( $\mathrm{Hp}$ ) was determined using the ribonuclease protection assay, western blotting, and immunofluorescence. Iron absorption was measured using radioactive ${ }^{59} \mathrm{Fe}$.

Correspondence to: GJ Anderson, Iron Metabolism Laboratory, Queensland Institute of Research, PO Royal Brisbane Hospital Brisbane Queensland 4029, Australia: gregA@qimr.edu.au Results: A decrease in intestinal iron absorption occurred following an oral dose of iron and this was associated with increased enterocyte iron levels, as assessed by iron regulatory protein activity and immunoblotting for ferritin. Reduced absorption was also accompanied by a rapid decrease in expression of the mRNAs encoding the brush border iron transport molecules Dcytb and the iron responsive element (IRE) containing the splice variant of DMT1. No such change was seen in expression of the non-IRE splice variant of DMT1 or the basolateral iron transport molecules Iregl and Hp. Similar changes were observed at the protein level.

Conclusions: These data indicate that brush border, but not basolateral, iron transport components are regulated locally by enterocyte iron levels and support the hypothesis that systemic stimuli exert their primary effect on basolateral transport molecules.

Accepted for publication

11 September 2002

I

ron is essential for almost all forms of life but is toxic in excess and therefore body iron levels must be tightly regulated. ${ }^{1-3}$ As the body cannot actively excrete iron, the body iron content must be regulated at the point of absorption across the mature villus enterocytes of the duodenum. ${ }^{4}$ The main stimuli for iron absorption are changes in body iron stores and the demands of the developing erythroid mass. ${ }^{5-7}$ Although the mechanism by which the intestine is signalled of these requirements remains elusive, it is known that these signals take $2-3$ days to influence absorption. ${ }^{8-10}$ It is widely accepted that this lag period represents the time taken for the immature crypt cells to migrate up the villus and differentiate and that factors influencing iron absorption programme the crypt cells to absorb the required amount of iron on maturity.

One aspect of regulation that does not conform with the 2-3 day lag period described above is the mucosal block phenomenon, which was first reported by Hahn et al in $1943^{8}$ and studied in more detail by Stewart et al in $1950 .{ }^{11}$ Both studies described the ability of a large oral dose of iron to reduce the absorption of a smaller dose administered several hours later Due to the short time interval between doses, the initial dose must be having a direct effect on the mature enterocytes rather than the cells of the crypts. Although being discounted as the mechanism by which iron absorption is regulated by body iron requirements, ${ }^{12} 13$ examination of the molecular basis of the mucosal block phenomenon may provide insight into this process. The recent identification of several of the key molecules involved in intestinal iron absorption now allows such studies to be undertaken.

The first mammalian iron transporter to be identified was divalent metal transporter l (DMT1), a brush border ferrous iron transport protein. ${ }^{14}{ }^{15}$ The mRNA transcript for this gene exists as at least two splice variants, one of which contains a putative iron responsive element (IRE). ${ }^{16}$ IREs are stem loop structures found in the non-coding region of various mRNA sequences and confer iron dependent regulation on these genes by virtue of their interactions with iron regulatory proteins (IRPs) (for review see Kühn ${ }^{17}$ ). The IRE containing splice variant of DMTI (DMTl(IRE)) is expressed at greater levels than the non-IRE splice variant (DMTI(non-IRE)) in the duodenum, ${ }^{1618}{ }^{19}$ suggesting a role for IRPs in the regulation of intestinal DMT1 expression. The physiological relevance of the IRE in DMTI(IRE) has yet to be determined but studies have demonstrated a rapid decrease in DMT1 message and protein following an oral dose of iron in rats. ${ }^{20}{ }^{21}$ Other more recently discovered molecules involved in intestinal iron absorption include the putative basolateral iron transporter Ireg $1,{ }^{22-24}$ the putative brush border ferrireductase Dcytb, ${ }^{25}$ and the intracellular ferroxidase hephaestin (Hp). ${ }^{19}{ }^{26}$ The IRE/IRP system is considered unlikely to play a role in the regulation of any of these molecules in the small intestine.

Although a potential role for IRPs and DMT1 in the mucosal block phenomenon has been reported, ${ }^{20} 21$ no investigation into the effect of an oral dose of iron on duodenal expression of Ireg l, Dcytb, or Hp has been carried out, nor have the relative contributions of the IRE and non-IRE splice variants of DMT1 been evaluated. In this study we conduct a detailed examination of expression of these genes and consider the importance of luminal iron in modulating the response of the intestine to variations in systemic iron requirements.

Abbreviations: $\beta \mathrm{m}, \beta$-mercaptoethanol; DMT1, divalent metal transporter 1; DMT 1 (IRE), IRE splice variant of DMT 1; DMT 1 (non-IRE), non-IRE splice variant of DMT1; GAPDH, glyceraldehyde-3-phosphate dehydrogenase; $\mathrm{Hp}$, hephaestin; IRE, iron responsive element; IRP, iron regulatory protein; RPA, ribonuclease protection assay. 


\section{METHODS}

\section{Animals and diets}

Newly weaned male Sprague-Dawley rats were used for all experiments. Rats were weaned onto an iron deficient diet (iron content $3 \mathrm{mg} / \mathrm{kg}$ wet weight), prepared as described by Valberg and colleagues. ${ }^{27}$ They were allowed unlimited access to food and deionised water and were maintained on this diet for two weeks. Rats were then given an intragastric dose of iron (10 $\mathrm{mg}$ for the time course and 0-20 $\mathrm{mg}$ for the dose-response) as $\mathrm{FeSO}_{4}$, in $250 \mu \mathrm{l} 10 \mathrm{mM} \mathrm{HCl}$ following an overnight fast. One hour after dosing, rats were returned to the iron deficient diet for the remainder of the experiment. At appropriate time points animals were anaesthetised (44 $\mathrm{mg} / \mathrm{kg}$ ketamine and $8 \mathrm{mg} / \mathrm{kg}$ xylazine), and duodenal enterocytes were isolated as previously described ${ }^{28}$ and snap frozen in liquid nitrogen. All experiments described in this study were approved by the Queensland Institute of Medical Research Animal Ethics Committee.

\section{Evaluation of iron absorption}

Iron uptake and transfer were determined in duplicate rats as follows. At appropriate time points, rats were anaesthetised as described above and a midline incision made in the abdomen. The duodenum was exposed and three loose ligatures tied around it: two approximately $0.5 \mathrm{~cm}$ distal to the pylorus and one at the ligament of Treitz. An incision was made proximal to the upper ligature, a cannula inserted, and the upper ligature tightened. A second incision was made distal to the lower ligature. The small intestine was flushed with approximately $5 \mathrm{ml}$ of saline (prewarmed to $37^{\circ} \mathrm{C}$ ) injected through the cannula. The lower ligature was then tightened to seal off the distal end of the duodenum. The duodenal loop was then infused with $100 \mu \mathrm{l}$ of $5 \mathrm{mM} \mathrm{HCl}$ containing $1.25 \mathrm{mg} / \mathrm{ml}(4.5$ $\mu \mathrm{M}) \mathrm{FeSO}_{4}$, and $10 \mu \mathrm{Ci} / \mathrm{ml}{ }^{59} \mathrm{Fe}$, and the residual solution flushed from the cannula with $150 \mu$ l saline. The second ligature at the proximal end of the loop was tightened and the cannula removed. The abdomen of the animal was covered in damp gauze to prevent drying and continually moistened with prewarmed saline.

Thirty minutes after administration of the test solution the animal was sacrificed by cervical dislocation and the duodenal loop carefully excised. The lumen was rinsed extensively with saline (approximately $30 \mathrm{ml}$ ) and the rinse solution retained. Radioactivity in the rinse solution, duodenum, and carcass was counted separately using a Ram DA Counter with PM-11 tube (Rotem Industries, Arava, Israel) at a distance of $10 \mathrm{~cm}$. Brush border iron uptake was calculated by adding the counts in the carcass and the duodenum and expressing this as a percentage of the total radioactivity injected. Basolateral transfer was represented by the counts in the carcass and was expressed as a percentage of uptake. Whole body iron absorption assays were performed for the dose-response and were carried out as previously described. ${ }^{19}$

\section{Ribonuclease protection assay}

Total RNA was extracted from tissue samples using Trizol reagent (Invitrogen, Melbourne, Australia) as per the manufacturer's instructions. Ribonuclease protection assays (RPAs) were performed as previously described ${ }^{19}$ using $5 \mu \mathrm{g}$ of total RNA. The housekeeping gene glyceraldehyde-3-phosphate dehydrogenase (GAPDH) was used for normalisation. The riboprobes used corresponded to the following cDNA sequences (the data presented in parentheses indicate section of cDNA and Genbank accession number): Hp (nt1360-1573, AF246120), Iregl (nt1190-1365, U76714), DMTl(IRE) (nt1413-1628, AF029757), DMTl(non-IRE) (nt1413-1690, AF029757), Dcytb (nt20-259, BF558425), GAPDH (nt536-691, AF 106860).

\section{IRP RNA binding assay}

Assays for IRP activity were performed as described below. A radiolabelled IRE containing riboprobe was synthesised as described above for the RPA using plasmid pGL66 which contains part of a rat L-ferritin pseudogene ${ }^{29}$ (kindly supplied by Dr Elizabeth Leibold). Before use, the probe was heated to $70^{\circ} \mathrm{C}$ for 10 minutes and allowed to cool to room temperature to allow the IRE to form. Protein was extracted from isolated duodenal cells by resuspending them in bandshift buffer ( 10 mM HEPES, 3 mM MgCl , 40 mM KCl, 5\% glycerol, 2 mM DTT, $\mathrm{pH} 7.5$ ) containing $0.5 \% \mathrm{NP}-40$ and protease inhibitors (Complete; Roche Molecular Biochemicals, Sydney, Australia). The solution was incubated on ice for 15 minutes and insoluble material was removed by centrifugation at full speed in a microfuge for 10 minutes at $4^{\circ} \mathrm{C}$. The protein concentration in the supernatant was determined using the Bio-Rad Protein Assay (Bio-Rad Laboratories, Hercules, California, USA).

Extracted protein $(0.5 \mu \mathrm{g})$ was incubated in bandshift buffer with $100000 \mathrm{cpm}$ of the riboprobe for 30 minutes at room temperature to allow binding of IRPs to the labelled IRE. Loading controls were prepared by adding $\beta$-mercaptoethanol $(\beta \mathrm{m})$ to a final concentration of $2 \%$ and incubating the samples for 10 minutes at room temperature prior to the addition of the riboprobe. This induces the full activity of IRPl by converting all of the protein to the RNA binding form. $\beta \mathrm{m}$ has no effect on IRP2 as this protein, unlike IRP1, has constitutive RNA binding activity and is regulated instead by protein degradation. ${ }^{30}$ Unbound riboprobe was digested with $1 \mathrm{U}$ of RNase Tl (Roche Molecular Biochemicals) for 10 minutes at room temperature. Heparin (Sigma-Aldrich, Castle Hill, Australia) was then added to a final concentration of $5 \mathrm{mg} / \mathrm{ml}$ and the solution incubated for a further 10 minutes at room temperature. Samples were mixed with $2 \mu \mathrm{l}$ of gel loading buffer (40\% sucrose, $0.25 \%$ bromophenol blue) and electrophoresed on a $6 \%$ non-denaturing TBE PAGE gel at $200 \mathrm{~V}$ for two hours. The gels were then dried and exposed to $x$ ray film.

\section{Antibody production}

Polyclonal antibodies to Hp, Iregl, and Dcytb were raised as previously described. ${ }^{19}{ }^{31}$ A DMTl antiserum recognising both the IRE and the non-IRE isoforms was raised in rabbits using the peptide CVKPSQSQVLRGMFV ${ }^{32}$ conjugated to diphtheria toxoid.

\section{Immunohistochemistry}

DMTl and Dcytb protein were localised by immunofluorescence microscopy using frozen sections cut from duodenal tissue snap frozen in OCT embedding compound (Sakura Finetek USA, Torrance, California, USA) as previously described. ${ }^{31}$

\section{Western blot analysis}

Protein was extracted from isolated enterocytes and expression of $\mathrm{Hp}$ and Iregl determined by western blotting as described previously. ${ }^{19} 31$ Ferritin expression was determined in the same fashion using a rabbit antiferritin antibody (ICN Biomedicals, Seven Hills, Australia) at 1 in 5000 dilution and using $100 \mu \mathrm{g}$ of protein sample. A rabbit antiactin antibody (Sigma-Aldrich) was used as a loading control.

\section{Statistical analysis}

All values are expressed as mean (SEM). Statistical differences between means were calculated with Microsoft Excel using the Student's $t$ test correcting for differences in sample variance.

\section{RESULTS}

Rats were treated with an intragastric $10 \mathrm{mg}$ blocking dose of iron before being administered a test dose of oral radioactive iron at appropriate time points. The percentage of this test dose transported across the brush border membrane (uptake) 


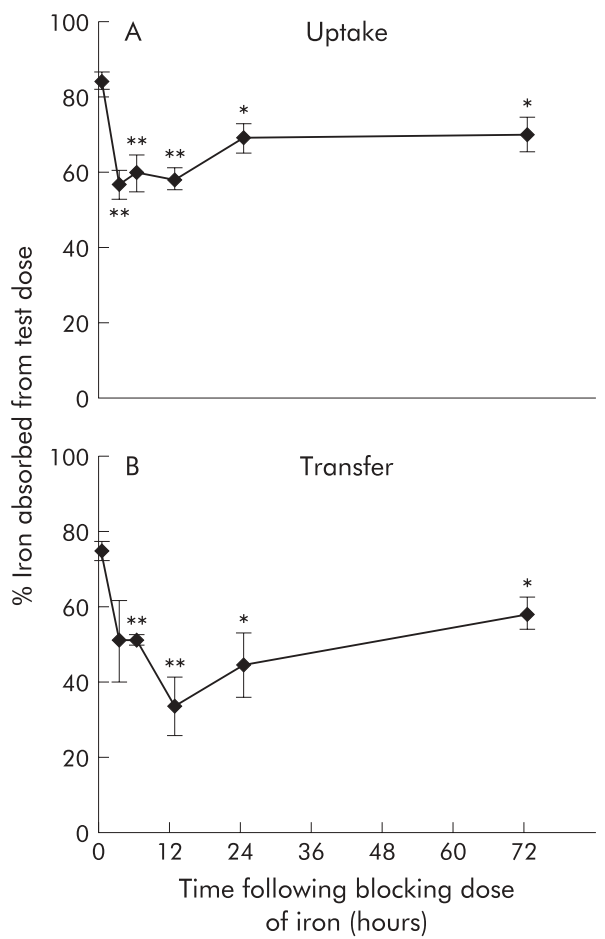

Figure 1 Duodenal uptake and transfer of ${ }^{59} \mathrm{Fe}$ in rats following a blocking dose of non-radioactive iron. Rats were fasted overnight before being administered $10 \mathrm{mg}$ of oral iron. At various time points thereafter, animals were anaesthetised and uptake $(\mathrm{A})$ and transfer (B) of ${ }^{59} \mathrm{Fe}$ determined as described in the methods section. The number of hours following the initial administration of iron is shown. Data are mean (SEM) of six animals. Statistical significance is shown relative to control animals: ${ }^{*} p<0.05,{ }^{* *} p<0.01$.

and the basolateral membrane (transfer) of the duodenal enterocytes is shown in fig l. Uptake across the brush border membrane decreased rapidly following a blocking dose of iron, from control levels of 84.6 (2.3)\% to a minimum of 57.1 (3.9)\% within three hours. Uptake persisted at this reduced level until the 12 hour time point before recovering to near control levels by 72 hours. Basolateral transfer followed a similar pattern, although it did not decline as rapidly, decreasing from a control value of $74.7(2.6) \%$ to $33.6(7.8) \%$ after 12 hours. Again, this was a transient decrease and transfer returned to near control levels by 72 hours.

Having confirmed that a mucosal block to absorption had occurred in the rats, IRP RNA binding activity in duodenal enterocytes was examined to provide a measure of the biologically accessible iron pool in these cells. Animals used in this study had been maintained on iron deficient diets prior to dosing with iron, and thus the enterocytes were expected to be iron deficient. This was confirmed by the high RNA binding activity of both IRPI and IRP2 in control animals (fig 2A). This decreased rapidly following the blocking dose of iron, with IRPI RNA binding activity substantially decreased after 30 minutes and IRP2 within one hour. There was minimal RNA binding activity for both IRP1 and IRP2 at the 6-24 hour time points, but by 72 hours almost complete recovery was seen for both proteins.

Binding of IRPs to IRE sequences affects expression of a number of iron metabolism genes. ${ }^{17}$ To ensure that the change in IRP RNA binding activity seen in this study could elicit normal changes in gene expression, the amount of ferritin protein in duodenal enterocytes was examined by western blotting (fig 2B). As would be expected in animals on an iron deficient diet, no detectable ferritin was found in control animals. Ferritin protein was first detected three hours after administration of iron, reaching a maximum at 12 hours. Again, the effect

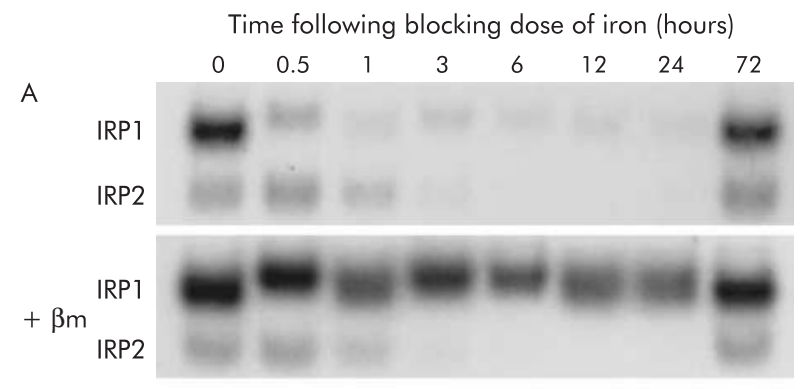

B

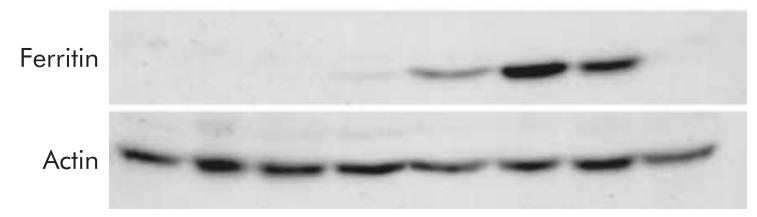

Figure 2 Iron regulatory protein (IRP) activity and ferritin expression in rats following orally administered iron. Rats were fasted overnight before being administered $10 \mathrm{mg}$ of oral iron. At various time points thereafter, animals were sacrificed and duodenal enterocytes isolated as described in the methods section. Protein was extracted from enterocytes and analysed by IRP bandshift assay (A) and by western blotting using antibodies specific for ferritin (B). Representative assays from triplicate experiments are shown. The number of hours following iron administration is indicated. $\beta \mathrm{m}$, $\beta$-mercaptoethanol.

was transient, with ferritin decreasing to undetectable levels by 72 hours.

To further investigate the molecular basis of the mucosal block phenomenon, expression of the genes proven or likely to be involved in iron absorption was determined using RPA analysis. Representative gels are shown in fig $3 \mathrm{~A}$ and quantitation in fig 3B. Expression of the brush border uptake components DMTI(IRE) and Dcytb decreased dramatically following an oral dose of iron. DMTI(IRE) expression was reduced to $28.8 \%$ of control levels by three hours and reached a minimum of $4.4 \%$ of control levels by 12 hours. The decrease in Dcytb message was slower and less pronounced, reaching $10.9 \%$ of control levels by 12 hours. Expression of both $D M T I(I R E)$ and Dcytb partially recovered to $51.0 \%$ and $55.7 \%$ of control levels, respectively, by 72 hours. No strong regulation of DMTI (non-IRE), Hp, or Iregl mRNAs was seen but a modest decrease in Iregl message to $56.9 \%$ of controls was recorded at 12 hours.

The changes in DMTI and Dcytb mRNA expression described above were confirmed at the protein level using immunofluorescence. Both DMTl and Dcytb protein were detected as strong fluorescence in the villus enterocytes of control animals (fig 4). This agrees with the published localisations for these two proteins. ${ }^{25} 3233$ Following an oral dose of iron, DMTl protein expression decreased to very low levels within six hours. Dcytb protein also declined considerably by 12 hours although some residual fluorescence remained on the brush border. Both proteins recovered to near control levels by 72 hours.

As there was very little change in Iregl or Hp message levels, any change in protein expression may be too subtle to be detected by immunofluorescence and therefore western blotting was employed to investigate this. As shown in fig 5, no change in expression of either protein was seen throughout the experiment.

To determine whether smaller doses of iron would produce smaller changes in both absorption and brush border gene expression, we carried out a dose-response study. For these experiments, all parameters were measured six hours after administration of iron as both absorption and gene expression had changed dramatically at this time in the studies described above. Absorption was determined using intact animals, as 

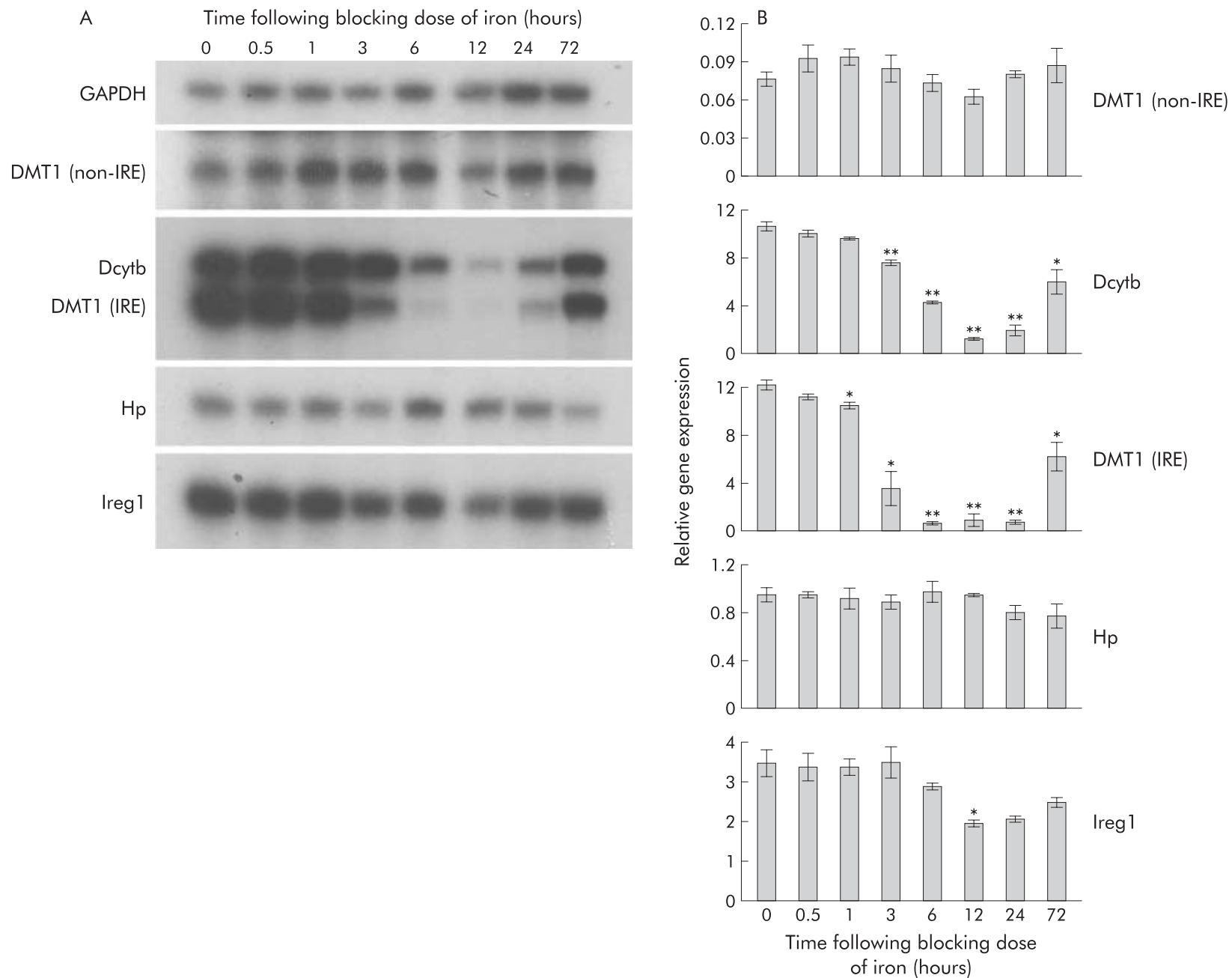

Figure 3 Gene expression following orally administered iron. Rats were fasted overnight before being administered $10 \mathrm{mg}$ of oral iron. At various time points thereafter, animals were sacrificed and duodenal enterocytes isolated as described in the methods section. Total RNA was extracted from enterocytes and gene expression determined by ribonuclease protection assay (RPA) using $5 \mu \mathrm{g}$ of RNA. Representative RPAs are shown for each gene (A). The number of hours following administration of iron is indicated. Band intensities were quantitated by densitometry, corrected for loading using glyceraldehyde-3-phosphate dehydrogenase (GAPDH) as a control, and graphed as a proportion of GAPDH (B). Data represent mean (SEM) of three animals. Statistical significance is shown relative to the 0 hour time point: * $p<0.05$, ${ }^{* *} p<0.01$. DMT1, divalent metal transporter 1; DMT1 (IRE), IRE splice variant of DMT1; DMT1 (non-IRE), non-IRE splice variant of DMT1; Hp, hephaestin; IRE, iron responsive element.

described in the methods section, and the results are shown in fig 6 . While a general trend towards decreasing absorption was seen with $0.5 \mathrm{mg}$ and $2 \mathrm{mg}$ of iron, statistical significance was not reached until a $10 \mathrm{mg}$ dose was used. A further decrease in absorption was observed using $20 \mathrm{mg}$ of iron. Examination of duodenal gene expression in these animals by RPA analysis revealed a progressive reduction in DMTI(IRE) and Dcytb expression (fig 7). Once again the decrease in Dcytb mRNA was not as dramatic as that seen for DMTl(IRE). There was little change in expression of DMTl (non-IRE), Hp, or Iregl messages.

\section{DISCUSSION}

Our understanding of the molecular basis of intestinal iron absorption has been greatly enhanced by the recent discoveries of several key molecules crucial for this process. By applying this knowledge to the mucosal block phenomenon, we have provided some significant insights into the regulation of this important physiological process. We have shown that the mucosal block to absorption is caused by a reduction in both the brush border uptake and basolateral transfer of iron in duodenal enterocytes and this is in agreement with previous studies. ${ }^{345}$ The decrease in brush border uptake appears to be due in part to a rapid decrease in expression of DMTl message and protein, again as reported previously. ${ }^{20}$ This study however is the first to show that the mucosal ferrireductase Dcytb is also rapidly affected by dietary iron. Our results indicate that the decrease in DMTI message is entirely due to a decrease in the level of the DMTl(IRE) isoform as there was no detectable decrease in DMTI (non-IRE). This, together with the corresponding decrease in IRP binding activity and increase in ferritin expression, supports the involvement of IRP/IRE interactions in the regulation of enterocyte DMTl expression. IRP/IRE interactions cannot directly explain the accompanying decrease in Dcytb message however, as mRNA does not contain an IRE motif. An alternative mechanism for the regulation of Dcytb message is also suggested by the slower and less pronounced decrease in expression that occurs following an oral dose of iron. Dcytb could be regulated at the transcriptional level, or its message could be under the control of a protein whose expression is IRP dependent, explaining the slightly delayed response. It is also possible that DMTl is under transcriptional control but recent studies using cultured cells suggest that this is not the case. ${ }^{36}$ Demonstration that changes in the expression of DMTl and Dcytb protein mimicked the changes in message level suggests that these proteins have a high rate of turnover and/or that they may be degraded in an iron dependent manner. 


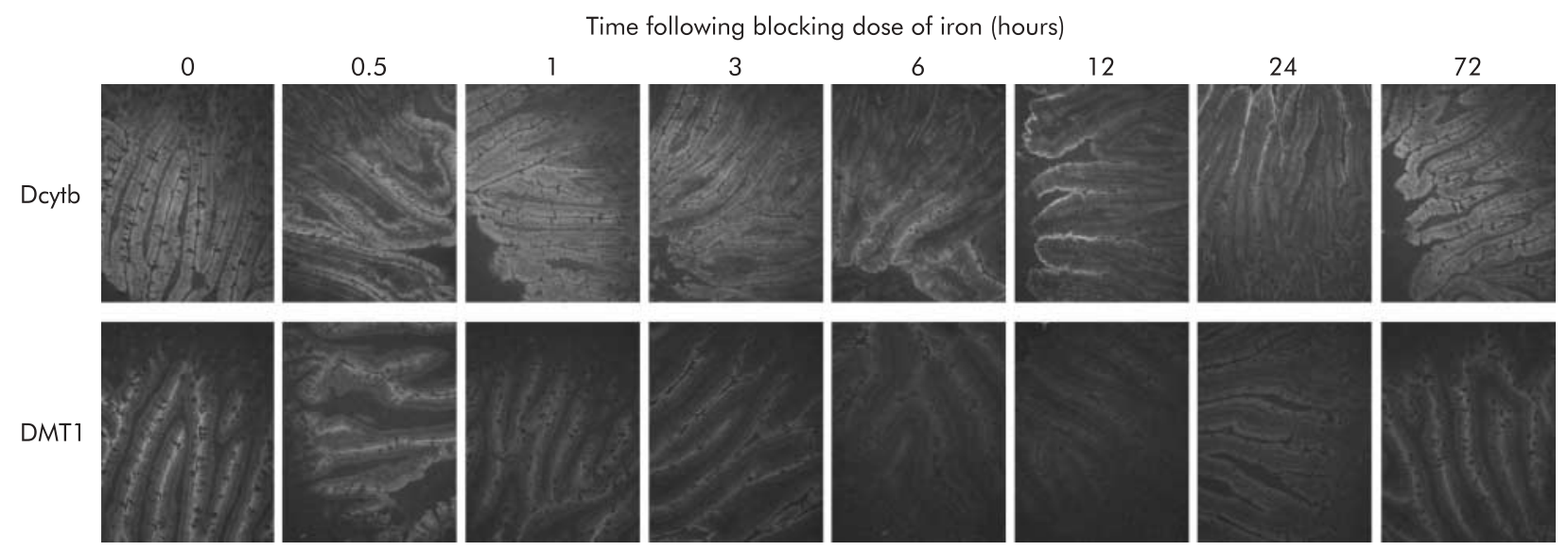

Figure 4 Immunofluorescence for divalent metal transporter 1 (DMT1) and Dcytb protein in the duodenum of rats following oral administration of iron. Rats were fasted overnight before being administered $10 \mathrm{mg}$ of oral iron. At various time points thereafter, animals were sacrificed and duodenal sections analysed by immunofluorescence using antibodies specific for DMT1 or Dcytb, as described in the methods section. Immunohistochemistry was carried out on sections from three animals and representative sections are shown. The number of hours following administration of iron is shown. Original magnification $200 \times$.

The observed decrease in iron export from enterocytes does not appear to involve a change in expression of the basolateral transporter Iregl or the intracellular ferroxidase $\mathrm{Hp}$ at the protein level. There are several possible explanations for this. The most likely explanation is the mixing of radioactive iron from the test dose with cold iron from the blocking dose that remained within the cell. This would lead to an apparent decrease in transfer due to a reduction in the specific activity of the intracellular iron available for basolateral export. The decrease in transfer may be exacerbated by increasing ferritin synthesis which reached a peak at 12 hours. This ferritin may sequester some of the iron entering the cell and impede its passage into the circulation. In time, the size of the enterocyte iron pool will decrease (as iron is moved across the basolateral membrane and cells are exfoliated) and this could explain the recovery of transfer at 24 hours despite high ferritin levels. An alternative explanation for the observed reduction in enterocyte iron export is a post-translational decrease in the activity of Ireg 1 and/or Hp. Whether these proteins can be regulated in this manner has not been investigated, but irrespective of this, it is not necessary to invoke such changes to explain the reduced transfer.

The studies described in this paper provide some fundamental insights into the relative contributions of luminal and systemic signals to the regulation of iron absorption. While expression of the brush border uptake components DMTI and Dcytb is regulated, at least in part, by intracellular iron levels, the basolateral transporter Iregl is not. However, we and others ${ }^{19}{ }^{24}$ have previously shown that Iregl expression can be regulated by the body's iron requirements, being increased in iron deficiency and decreased in iron loading. These observations suggest that basolateral transfer is regulated by signals

\section{A}

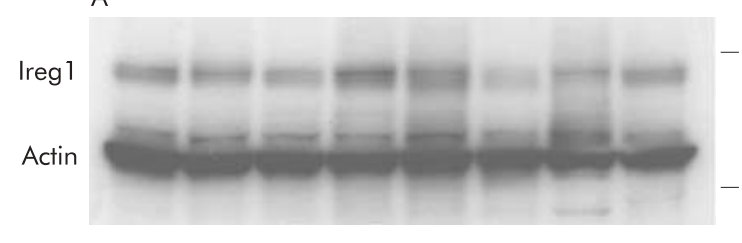

85

$-41.7$
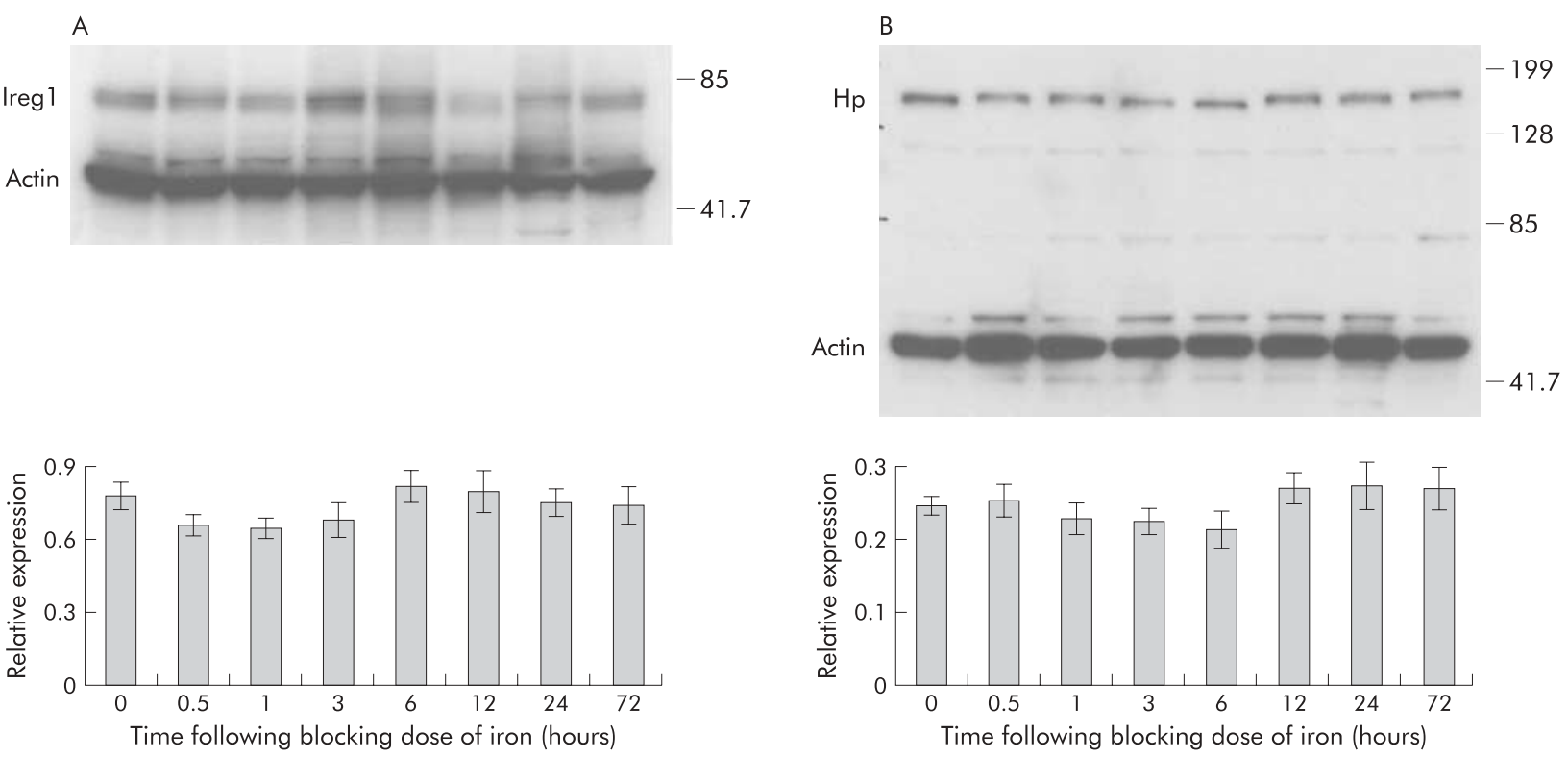

Figure 5 Detection of Ireg 1 and hephaestin $(\mathrm{Hp}$ ) protein in the duodenum of rats following oral administration of iron. Rats were fasted overnight before being administered $10 \mathrm{mg}$ of oral iron. At various time points thereafter, animals were sacrificed and duodenal enterocytes isolated, as described in the methods section. Protein was extracted from enterocytes and analysed by western blotting using antibodies specific for Iregl (A) or $\mathrm{Hp}$ (B). Representative blots are shown for each protein. The number of hours following iron administration is indicated. The sizes $\left(\times 10^{-3}\right)$ of the molecular weight standards are indicated on the right of each blot. Band intensities were quantitated by densitometry, corrected for loading using actin as a control, and graphed as a proportion of actin. Data are mean (SEM) of 5-6 animals. No statistically significant differences were seen between the groups. 


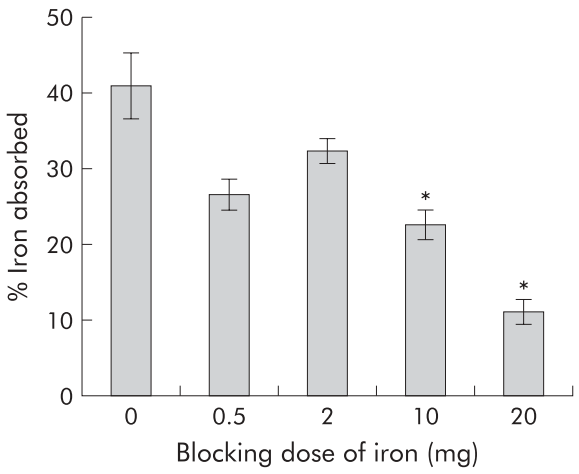

Figure 6 Iron absorption following varying doses of iron. Rats were fasted overnight before being administered an oral dose containing varying amounts of iron. Control animals were given $10 \mathrm{mM} \mathrm{HCl}$ only. Intestinal iron absorption was determined using an oral dose of ${ }^{59} \mathrm{Fe}$ administered six hours after the initial dose of iron. Absorption is presented as the percentage of radioactivity retained by the animals five days after dosing. The amount of iron received by each group is indicated (blocking dose of iron). Data are mean (SEM) of three animals. Statistical significance is shown relative to control animals: ${ }^{*} p<0.05$.

from the body rather than by the local iron concentration and support our earlier hypothesis that basolateral transfer is the primary point at which intestinal iron absorption is regulated.$^{37}$ In this hypothesis, signals to alter iron absorption are detected by the intestinal epithelium and result in changes in expression of Iregl. For example, an increase in Iregl expression would lead to increased basolateral transfer and a decrease in enterocyte iron levels. This in turn would lead to an increase in IRP RNA binding activity, stabilising DMTI (IRE) mRNA and increasing DMTl protein expression. The decrease in intracellular iron level would also increase Dcytb expression. The net result would be an increase in brush border iron uptake subsequent to an increase in basolateral transfer. The opposite would occur when iron requirements are low and Ireg l expression is reduced. Iron would build up within the enterocyte, reducing IRP activity and subsequently decreasing DMTl and Dcytb expression and, consequently, brush border iron uptake. It is possible (if not likely) that DMTl and Dcytb levels are also modulated by systemic influences on enterocytes, but importantly expression of these molecules can be overridden by local regulation that does not affect Iregl.

The rapid changes in DMTl and Dcytb expression in response to intracellular iron may be a mechanism by which a constant supply of iron can be provided to the basolateral transfer mechanism regardless of dietary iron content. If the amount of dietary iron entering the cell exceeds the capacity of the basolateral transport pathway, iron will accumulate in the enterocyte leading to a reduction in expression of brush border iron transport components, which in turn will prevent a further build up of iron within the cell. If dietary intake is insufficient to meet body demands however, DMTl and Dcytb expression can be dramatically increased to extract as much iron as possible from an iron deficient diet. This is evident from the dose-response study in which iron absorption did not change significantly with doses of $0.5 \mathrm{mg}$ and $2 \mathrm{mg}$ of iron despite large decreases in DMTI(IRE) and Dcytb mRNA expression. Such data show that under iron deficient conditions expression of DMTl and Dcytb is far in excess of that necessary to adequately supply the basolateral transport mechanism.

In summary, our results indicate that brush border iron uptake is most strongly regulated by intracellular iron levels and support the proposal that basolateral transfer is regulated by systemic factors which reflect body iron requirements. The nature of these body signals is unclear but the recently identi-

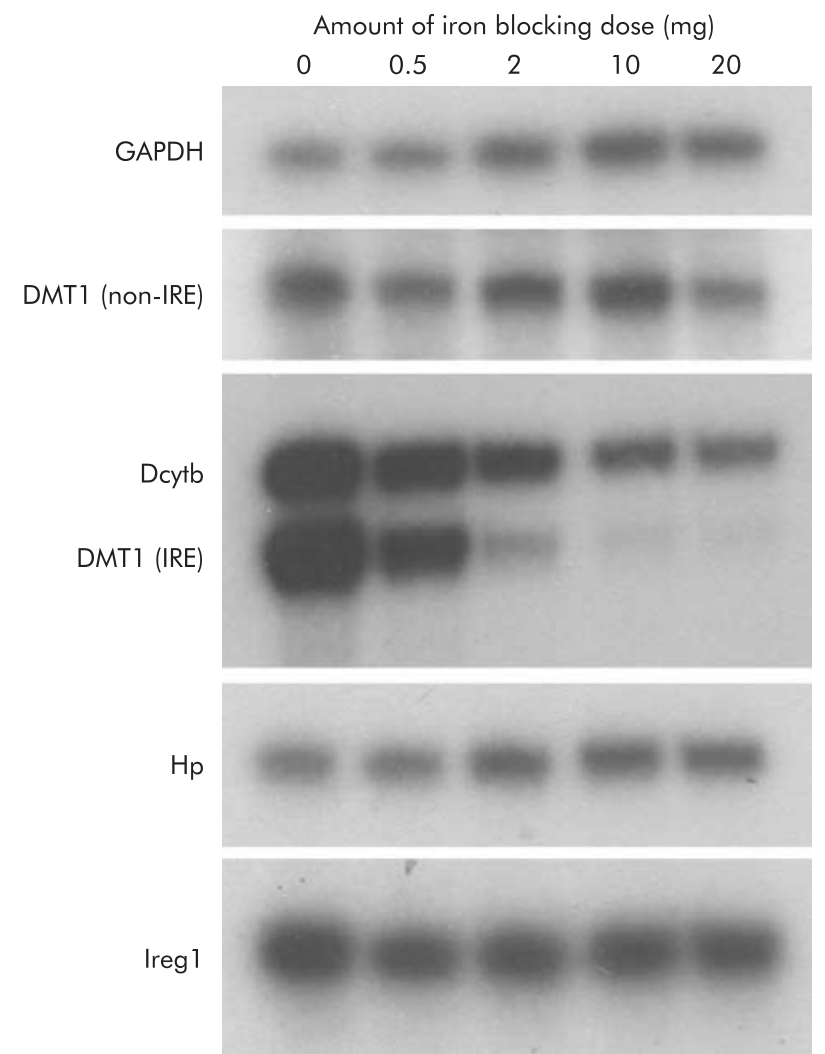

Figure 7 Gene expression following varying doses of oral iron. Rats were fasted overnight before being administered an oral dose containing varying amounts of iron. Control animals were given 10 $\mathrm{mM} \mathrm{HCl}$ only. Six hours after this dose, animals were sacrificed and duodenal enterocytes isolated, as described in the methods section. Total RNA was extracted from enterocytes and gene expression determined by ribonuclease protection assay using $5 \mathrm{\mu g}$ of RNA. Representative assays from triplicate experiments are shown. The amount of iron received by each group is indicated (iron blocking dose). DMT1, divalent metal transporter 1; DMT1 (IRE), IRE splice variant of DMT 1; DMT1 (non-IRE), non-IRE splice variant of DMT1; $\mathrm{Hp}$, hephaestin; IRE, iron responsive element.

fied hepcidin molecule may provide some of the answers. It is proposed that circulating hepcidin signals the body's iron requirements to the cells of the intestine ${ }^{38}$ and we have recently reported an exquisite inverse relationship between hepcidin expression in the liver and iron transporter expression in the duodenum..$^{31}$ The studies presented here suggest that a primary target of hepcidin may be Ireg $\mathrm{l}$.

\section{ACKNOWLEDGEMENTS}

We thank Dr Elizabeth Leibold for providing the pGL66 plasmid. DMF was supported by a postgraduate scholarship from the Royal Children's Hospital Foundation, Brisbane, Australia. This work was supported in part by grants from the National Health and Medical Research Council of Australia (to GJA), from the National Institute of Diabetes and Digestive and Kidney Diseases (R01 DK-57800-1 to CDV, GJA, and ATM), and from the Human Frontier Science Program (grant RGY0328/2001-M to ATM, CDV, and GJA).

\section{Authors' affiliations}

D M Frazer, S J Wilkins, E M Becker, T L Murphy, G J Anderson, Joint Clinical Sciences Program, the Queensland Institute of Medical Research and the University of Queensland, PO Royal Brisbane Hospital, Brisbane, Queensland 4029, Australia

C D Vulpe, Department of Nutritional Sciences, University of California, Berkeley, Berkeley CA 94720, USA

A T McKie, Division of Life Sciences, King's College, London SE 1 9NN, UK 


\section{REFERENCES}

1 Conrad ME, Barton JC. Factors affecting iron balance. Am J Hematol $1981 ; 10: 199-225$.

2 Crichton RR, Charloteaux-Wauters M. Iron storage and transport. Eur J Biochem 1987; 164:485-506.

3 Lombard M, Chua E, O'Toole P. Regulation of intestinal non-haem iron absorption. Gut 1997;40:435-9.

4 McCance RA, Widdowson EM. Absorption and excretion of iron. Lancet 1937;ii:680-4

5 Bothwell TH, Pirzio-Biroli G, Finch CA. Iron absorption I. Factors influencing absorption. J Lab Clin Med 1958;51:24-36.

6 Conrad ME, Foy AL, Williams HL, et al. Effect of starvation and protein depletion on ferrokinetics and iron absorption. Am J Physiol 1967; 213:557-65.

7 Mattii R, Mielke $\mathrm{CH}$, Levine $\mathrm{PH}$, et al. Iron in the duodenal mucosa of normal, iron- loaded and iron-deficient rats. Blood 1973:42:959-66. 8 Hahn PF, Bale WF, Ross JF, et al. Radioactive iron absorption by Hahn PF, Bale WF, Ross JF, et al. Radioactive iron absorption by
gastro-intestinal tract: influence of anemia, anoxia, and antecedent gastro-intestinal tract: influence of anemia, anoxia, and antecedent
feeding distribution in growing dogs. J Exp Med 1943;78:169-88.

9 Weintraub LR, Conrad ME, Crosby WH. The significance of iron turnover in the control of iron absorption. Blood 1964;24:19-24.

10 Linder MC, Munro HN. The mechanism of iron absorption and its regulation. Fed Proc 1977;36:2017-23.

11 Stewart WB, Yuile CL, Claiborne HA, et al. Radioiron absorption in anemic dogs: fluctuations in the mucosal block and evidence for a gradient of absorption in the gastrointestinal tract. J Exp Med 1950;92:375-82

12 Brown EB, Dubach R, Moore CV. Studies in iron transportation and metabolism XI. Critical analysis of mucosal block by large doses of inorganic iron in human subjects. J Lab Clin Med 1958;52:335-55.

13 Crosby WH. The control of iron balance by the intestinal mucosa. Blood 1963;22:441-9.

14 Fleming MD, Trenor III CC, Su MA, et al. Microcytic anaemia mice have a mutation in Nramp2, a candidate iron transporter gene. Nat Genet 1997:16:383-6.

15 Gunshin H, Mackenzie B, Berger UV, et al. Cloning and characterization of a mammalian proton-coupled metal-ion transporter. Nature 1997;388:482-8.

16 Lee PL, Gelbart T, West C, et al. The human Nramp2 gene: characterization of the gene structure, alternative splicing, promotor region and polymorphisms. Blood Cells Mol Dis 1998;24:199-215.

17 Kühn LC. Iron and gene expression: molecular mechanisms regulating cellular iron homeostasis. Nutr Revs 1998;56:S1 1-19.

18 Fleming RE, Migas MC, Zhou XY, et al. Mechanism of increased iron absorption in murine model of hereditary hemochromatosis: increased duodenal expression of the iron transporter DMT1. Proc Natl Acad Sci U S A 1999;96:3143-8.

19 Frazer DM, Vulpe CD, McKie AT, et al. Cloning and gastrointestinal expression of rat hephaestin: relationship to other iron transport proteins. Am J Physiol 2001;281:G931-9.
20 Oates PS, Trinder D, Morgan EH. Gastrointestinal function, divalent metal transporter-1 expression and intestinal iron absorption. Pflügers Arch 2000:440:496-502.

21 Yeh K, Yeh M, Watkins JA, et al. Dietary iron induces rapid changes in rat intestinal divalent metal transporter expression. Am J Physiol 2000;279:G1070-9.

22 Donovan A, Brownlie A, Zhou Y, et al. Postional cloning of zebrafish ferroportin 1 identifies a conserved vertebrate iron exporter. Nature 2000;403:776-81.

23 McKie AT, Marciani P, Rolfs A, et al. A novel duodenal iron-regulated transporter, IREG 1, implicated in the basolateral transfer of iron to the circulation. Mol Cell 2000;5:299-309.

24 Abboud S, Haile DJ. A novel mammalian iron-regulated protein involved in intracellular iron metabolism. J Biol Chem 2000;275:19906-12.

25 McKie AT, Barrow D, Latunde-Dada GO, et al. An iron-regulated ferric reductase associated with the absorption of dietary iron. Science 2001;291:1755-9.

26 Vulpe CD, Kuo Y, Murphy TL, et al. Hephaestin, a ceruloplasmin homologue implicated in intestinal iron transport, is defective in the sla mouse. Nat Genet 1999:21:195-9.

27 Valberg LS, Taylor KB, Witts $L$, et al. The effect of iron-deficiency on the stomach of the rat. Br J Nutr 1961;15:473-80.

28 Anderson GJ, Powell LW, Halliday JW. The endocytosis of transferrin by rat intestinal epithelial cells. Gastroenterology 1994;106:414-22.

29 Leibold EA, Munro HN. Cytoplasmic protein binds in vitro to a highly conserved sequence in the $5^{\prime}$ untranslated region of ferritin heavy-and light-subunit mRNAs. Proc Natl Acad Sci U S A 1988;85:2171-5.

30 Henderson BR, Kühn LC. Differential modulation of the RNA-binding proteins IRP-1 and IRP-2 in response to iron: IRP-2 inactivation requires translation of another protein. J Biol Chem 1995;270:20509-15

31 Frazer DM, Wilkins SJ, Becker EM, et al. Hepcidin expression inversely correlates with the expression of duodenal iron transporters and iron absorption in rats. Gastroenterology 2002;123:835-44.

32 Trinder D, Oates PS, Thomas $C$, et al. Localisation of divalent metal transporter 1 (DMT1) to the microvillus membrane of rat duodenal enterocytes in iron deficiency, but to hepatocytes in iron overload. Gut 2000:46:270-6.

33 Canonne-Hergaux F, Gruenheid S, Ponka P, et al. Cellular and subcellular localization of the Nramp2 iron transporter in the intestinal brush border and regulation by dietary iron. Blood 1999;93:4406-17.

34 Manis JG Schachter D. Active transport of iron by intestine: effects of oral iron and pregnancy. Am J Physiol 1962;203:81-6.

35 Wheby MS, Jones LG, Crosby WH. Studies on iron absorption. Intestinal regulatory mechanisms. J Clin Invest 1964;43:1433-42.

36 Gunshin H, Allerson CR, Polycarpou-Schwarz M, et al. Iron-dependent regulation of the divalent metal ion transporter. FEBS Lett 2001;509:309-16.

37 Frazer DM, Anderson GJ. Intestinal iron transport and its regulation. Hematology 2001:6:193-203.

38 Nicolas G, Bennoun M, Devaux I, et al. Lack of hepcidin gene expression and severe tissue iron overload in upstream stimulatory factor 2 (USF2) knockout mice. Proc Natl Acad Sci U S A 2001;98:8780-5. 